\title{
NNNLO soft-gluon corrections for the top-antitop pair production cross section
}

\author{
Nikolaos Kidonakis \\ Kennesaw State University, Physics \#1202, \\ Kennesaw, GA 30144, USA
}

\begin{abstract}
I present a calculation of next-to-next-to-next-to-leading-order (NNNLO) soft-gluon corrections for top-antitop pair production in hadronic collisions. Approximate NNNLO (aNNNLO) results are obtained by adding the NNNLO soft-gluon corrections to the complete next-to-next-to-leading-order (NNLO) cross section. Theoretical predictions are shown for the total aNNNLO $t \bar{t}$ cross section at LHC and Tevatron energies. The aNNNLO cross sections are larger but have smaller theoretical uncertainties than at NNLO.
\end{abstract}

\section{Introduction}

The top quark retains a leading position among elementary particles as the heaviest particle that has been discovered so far. Top-quark physics occupies a prominent role in the LHC program as well as past experiments at the Tevatron. Top quark studies, both in top-antitop pair production and in single-top production, are important for QCD and electroweak physics, and many measurements of total cross sections, differential distributions, and top quark properties have been performed at the Tevatron and the LHC. The large mass of the top quark suggests that it plays an important role in Higgs physics. The top quark also figures prominently in searches for flavor-changing neutral currents and in searches for other physics beyond the Standard Model.

The experimental measurements of the top quark total cross section, transverse momentum $\left(p_{T}\right)$ distributions, and rapidity distributions, are currently in good agreement with theoretical predictions. The experimental errors continue to decrease with time, which necessitates increasingly precise theoretical calculations. Next-to-leading order (NLO) calculations of the $t \bar{t}$ cross section have been available for a long time [1, 2, 3. The NLO predictions have been improved by the inclusion of higher-order soft-gluon corrections through resummations, first at next-to-leading-logarithm (NLL) accuracy and, more recently, at next-to-next-to-leadinglogarithm (NNLL) accuracy. A brief history of these soft-gluon threshold resummations and a large list of references can be found in the review paper of Ref. [4]. Approximate NNLO results have also been obtained from those resummations. In addition a large effort has been invested in the calculation of complete NNLO corrections (see again [4] for more details and references). At present there is still not a complete analytical calculation at NNLO, but numerical results for the total $t \bar{t}$ cross section at NNLO appeared in [5].

There are many differences between various resummation approaches in the literature and these have been detailed previously in [4]. Some resummations are for double-differential cross sections while others are only for total cross sections; some resummation formalisms 
use moment-space perturbative QCD (pQCD) while others use Soft-Collinear Effective Theory (SCET). There are also additional technical differences among the various methods [4].

The double-differential resummation approach uses a more general definition of threshold and it allows the calculation of transverse momentum and rapidity distributions, in addition to total cross-section calculations. In Ref. [6] NNLL resummation for the double-differential cross section using moment-space pQCD was used to derive approximate NNLO results for the total $t \bar{t}$ cross section and top-quark differential distributions. Now that exact NNLO numerical results for the total cross section are available, one can improve the theoretical prediction for that quantity by adding aNNNLO corrections from soft-gluon resummation.

The validity, stability, and reliability of the theoretical predictions from our resummation formalism has been discussed several times before, most recently in Ref. [7]. The NLO and NNLO soft-gluon corrections from our formalism in Refs. [6, 7] are very close to the exact results. The numerical accuracy of the threshold soft-gluon approximation was first demonstrated by comparing exact NLO and approximate NLO results for the total $t \bar{t}$ cross section and the top quark $p_{T}$ and rapidity distributions. If one compares the total NLO cross section the difference between approximate and exact results is entirely negligible, well below 1\%. This excellent agreement between exact and approximate NLO results is true not only for the total cross section but also for the differential distributions (see Fig. 11 and related discussion in [7]).

We can also make comparisons at NNLO for the total $t \bar{t}$ cross section. Our approximate NNLO cross sections [6, 7] are very close to the exact NNLO [5] results: both the central values and the scale uncertainty are nearly the same and this holds true for all LHC and Tevatron collider energies and top quark masses (see Figs. 13 and 14 and the corresponding discussion in [7]). There is less than $1 \%$ difference between approximate and exact cross sections at both NLO and NNLO, and our results approximate the exact results better than other methods such as the one used in [5]. This is a very important fact since it displays the validity and relevance of our method and it provides strong confidence that the aNNNLO soft-gluon corrections will be good approximations to the exact results at that higher order. Also the comparison of our results with Tevatron and LHC total cross sections and $p_{T}$ and rapidity distributions shows excellent agreement between our theoretical predictions and the experimental data (see e.g. Ref. [7]).

In the next section we give some more details of our calculation and present analytical results for the aNNNLO corrections. Section 3 includes all our numerical results for the $t \bar{t}$ total cross section at $1.96 \mathrm{TeV}$ Tevatron energy and at 7, 8, 13, and 14 LHC energies. Our aNNNLO predictions are found by adding the NNNLO soft-gluon corrections to the complete NNLO $t \bar{t}$ total cross section. We conclude in Section 4.

\section{Analytical results}

Soft-gluon resummation follows from the renormalization group evolution of functions describing collinear and soft-gluon emission in the factorized partonic cross section, and it involves the details of the color structure of the process. The resummed cross section can be expanded at fixed order in the strong coupling, $\alpha_{s}$, to NLO, NNLO, NNNLO, etc., and inverted back to 
momentum space. The necessary ingredients for our calculation can be found in Refs. [6, 7, 8].

At leading order (LO) the two partonic channels for $t \bar{t}$ production are quark-antiquark annihilation

$$
q\left(p_{1}\right)+\bar{q}\left(p_{2}\right) \rightarrow t\left(p_{3}\right)+\bar{t}\left(p_{4}\right)
$$

and gluon-gluon fusion

$$
g\left(p_{1}\right)+g\left(p_{2}\right) \rightarrow t\left(p_{3}\right)+\bar{t}\left(p_{4}\right)
$$

We define the kinematical variables $s=\left(p_{1}+p_{2}\right)^{2}, t_{1}=\left(p_{1}-p_{3}\right)^{2}-m_{t}^{2}, u_{1}=\left(p_{2}-p_{3}\right)^{2}-m_{t}^{2}$, and $\beta=\sqrt{1-4 m_{t}^{2} / s}$, where $\beta$ is the top-quark speed in LO kinematics and $m_{t}$ is the top-quark mass. We also define the threshold variable $s_{4}=s+t_{1}+u_{1}$. At partonic threshold there is no energy available for additional radiation and $s_{4}$ vanishes in that limit. It is important to note that partonic threshold is a more general concept than absolute threshold, the latter meaning that the top quark is produced at rest. In other words if $s_{4}=0$ that does not mean that $\beta$ has to be zero; partonic threshold can be reached even with arbitrarily high transverse momentum of the top quark. In our resummation the threshold limit is always given by $s_{4} \rightarrow 0$.

At each order in $\alpha_{s}$, one encounters plus-distribution terms of the form $\left[\ln ^{k}\left(s_{4} / m_{t}^{2}\right) / s_{4}\right]_{+}$ and, for the $n$-th order corrections, the power of the logarithm, $k$, can range from the leading value of $2 n-1$ down to the lowest value of 0 . Thus, at NLO the leading value of $k$ is 1 , at NNLO it is 3 , and at NNNLO it is 5 . The expressions for the NNNLO soft-gluon corrections fully written out are extremely long, although they can be written in a more compact implicit form as first shown in [8]. However, the first two powers of the logarithms have coefficients that are relatively short and we display them below.

For $q \bar{q} \rightarrow t \bar{t}$, the NNNLO soft-gluon corrections to the double-differential cross section are

$$
\begin{aligned}
\frac{d^{2} \sigma_{q \bar{q} \rightarrow t \bar{t}}^{(3)}=}{d t_{1} d u_{1}}=\frac{\alpha_{s}^{5}}{\pi^{2}} & \frac{C_{F}}{N_{c} s^{2}}\left(\frac{t_{1}^{2}+u_{1}^{2}}{s^{2}}+\frac{2 m_{t}^{2}}{s}\right)\left\{8 C_{F}^{3}\left[\frac{\ln ^{5}\left(s_{4} / m_{t}^{2}\right)}{s_{4}}\right]_{+}\right. \\
+ & {\left[20 C_{F}^{3}\left(4 \ln \left(\frac{t_{1}}{u_{1}}\right)-\ln \left(\frac{t_{1} u_{1}}{m_{t}^{4}}\right)-\ln \left(\frac{\mu_{F}^{2}}{s}\right)-\frac{\left(1+\beta^{2}\right)}{2 \beta} \ln \left(\frac{1-\beta}{1+\beta}\right)-1\right)\right.} \\
& +10 C_{F}^{2} C_{A}\left(-3 \ln \left(\frac{t_{1}}{u_{1}}\right)+\ln \left(\frac{t_{1} u_{1}}{s m_{t}^{2}}\right)+\frac{\left(1+\beta^{2}\right)}{2 \beta} \ln \left(\frac{1-\beta}{1+\beta}\right)-\frac{11}{9}\right) \\
& \left.\left.+\frac{20}{9} C_{F}^{2} n_{f}\right]\left[\frac{\ln ^{4}\left(s_{4} / m_{t}^{2}\right)}{s_{4}}\right]_{+}\right\} \\
+ & \cdots
\end{aligned}
$$

where $C_{F}=\left(N_{c}^{2}-1\right) /\left(2 N_{c}\right)$, with $N_{c}=3$ the number of colors, $C_{A}=N_{c}$, and $n_{f}=5$ is the number of light-quark flavors. As discussed above, we do not display cubic or lower logarithmic powers, which have very long expressions for their coefficients.

For $g g \rightarrow t \bar{t}$, the NNNLO soft-gluon corrections to the double-differential cross section are

$$
\begin{aligned}
\frac{d^{2} \sigma_{g g \rightarrow t \bar{t}}^{(3)}}{d t_{1} d u_{1}}= & \frac{\alpha_{s}^{5}}{\pi^{2}} \frac{2 N_{c} C_{F}}{\left(N_{c}^{2}-1\right)^{2} s^{2}}\left(C_{F}-C_{A} \frac{t_{1} u_{1}}{s^{2}}\right)\left[\frac{t_{1}}{u_{1}}+\frac{u_{1}}{t_{1}}+\frac{4 m_{t}^{2} s}{t_{1} u_{1}}\left(1-\frac{m_{t}^{2} s}{t_{1} u_{1}}\right)\right] \\
& \times\left\{8 C_{A}^{3}\left[\frac{\ln ^{5}\left(s_{4} / m_{t}^{2}\right)}{s_{4}}\right]_{+}\right.
\end{aligned}
$$




$$
\begin{aligned}
& \left.\quad+\left[-20 C_{A}^{3}\left(\ln \left(\frac{t_{1} u_{1}}{m_{t}^{4}}\right)+\frac{11}{18}\right)-20 C_{A}^{2} C_{F} \ln \left(\frac{\mu_{F}^{2}}{s}\right)+\frac{20}{9} C_{A}^{2} n_{f}\right]\left[\frac{\ln ^{4}\left(s_{4} / m_{t}^{2}\right)}{s_{4}}\right]_{+}\right\} \\
& +\frac{\alpha_{s}^{5}}{\pi^{2}} \frac{10 C_{A}^{2}}{\left(N_{c}^{2}-1\right) s^{2}}\left[\frac{t_{1}}{u_{1}}+\frac{u_{1}}{t_{1}}+\frac{4 m_{t}^{2} s}{t_{1} u_{1}}\left(1-\frac{m_{t}^{2} s}{t_{1} u_{1}}\right)\right] \\
& \times\left\{N_{c}\left(1-\frac{2 t_{1} u_{1}}{s^{2}}\right)\left[\left(-C_{F}+\frac{C_{A}}{2}\right) \frac{\left(1+\beta^{2}\right)}{2 \beta} \ln \left(\frac{1-\beta}{1+\beta}\right)-C_{F}+\frac{N_{c}}{2} \ln \left(\frac{t_{1} u_{1}}{m_{t}^{2} s}\right)\right]\right. \\
& \quad+\frac{1}{N_{c}}\left(C_{F}-C_{A}\right) \frac{\left(1+\beta^{2}\right)}{2 \beta} \ln \left(\frac{1-\beta}{1+\beta}\right)+\frac{C_{F}}{C_{A}}-\ln \left(\frac{t_{1} u_{1}}{m_{t}^{2} s}\right) \\
& \left.\quad+\frac{N_{c}^{2}}{2} \frac{\left(t_{1}^{2}-u_{1}^{2}\right)}{s^{2}} \ln \left(\frac{u_{1}}{t_{1}}\right)\right\}\left[\frac{\ln ^{4}\left(s_{4} / m_{t}^{2}\right)}{s_{4}}\right]_{+} \\
& +\cdots
\end{aligned}
$$

where again we have only explicitly shown the first two powers of the logarithms.

We employ the above analytical results to calculate the aNNNLO $t \bar{t}$ cross sections at the LHC and the Tevatron in the next section.

\section{Total $t \bar{t}$ cross sections at the LHC and the Tevatron}

We now provide a study of the top-antitop pair production cross sections at the Tevatron and LHC colliders. We present approximate NNNLO (aNNNLO) calculations for these quantities. The aNNNLO results are computed by adding the NNNLO soft-gluon corrections (derived from NNLL resummation) to the exact NNLO quantities. We use the MSTW2008 NNLO parton distibution functions (pdf) [9].

\begin{tabular}{|c|c|c|c|c|c|}
\hline \multicolumn{7}{|c|}{ aNNNLO $t \bar{t}$ cross section $(\mathrm{pb})$} \\
\hline$m_{t}(\mathrm{GeV})$ & Tevatron & LHC 7 TeV & LHC 8 TeV & LHC $13 \mathrm{TeV}$ & LHC $14 \mathrm{TeV}$ \\
\hline 170 & 8.18 & 192 & 273 & 886 & 1046 \\
\hline 171 & 7.92 & 186 & 265 & 862 & 1018 \\
\hline 172 & 7.67 & 181 & 257 & 839 & 991 \\
\hline 173 & 7.44 & 175 & 250 & 816 & 964 \\
\hline 174 & 7.20 & 170 & 243 & 794 & 939 \\
\hline 175 & 6.98 & 165 & 236 & 773 & 914 \\
\hline
\end{tabular}

Table 1: The aNNNLO $t \bar{t}$ production cross section in pb in $p \bar{p}$ collisions at the Tevatron with $\sqrt{S}=1.96 \mathrm{TeV}$ and in $p p$ collisions at the $\mathrm{LHC}$ with $\sqrt{S}=7,8,13$, and $14 \mathrm{TeV}$. We set $\mu=m_{t}$ and use the MSTW2008 NNLO pdf [9].

Table 1 lists the central values for the aNNNLO $t \bar{t}$ cross sections at Tevatron and LHC energies for top quark masses between $170 \mathrm{GeV}$ and $175 \mathrm{GeV}$. For these central values we set the factorization and renormalization scales $\mu=m_{t}$ and we use the central pdf set of [9]. There are two kinds of theoretical uncertainties associated with the calculation: dependence on 


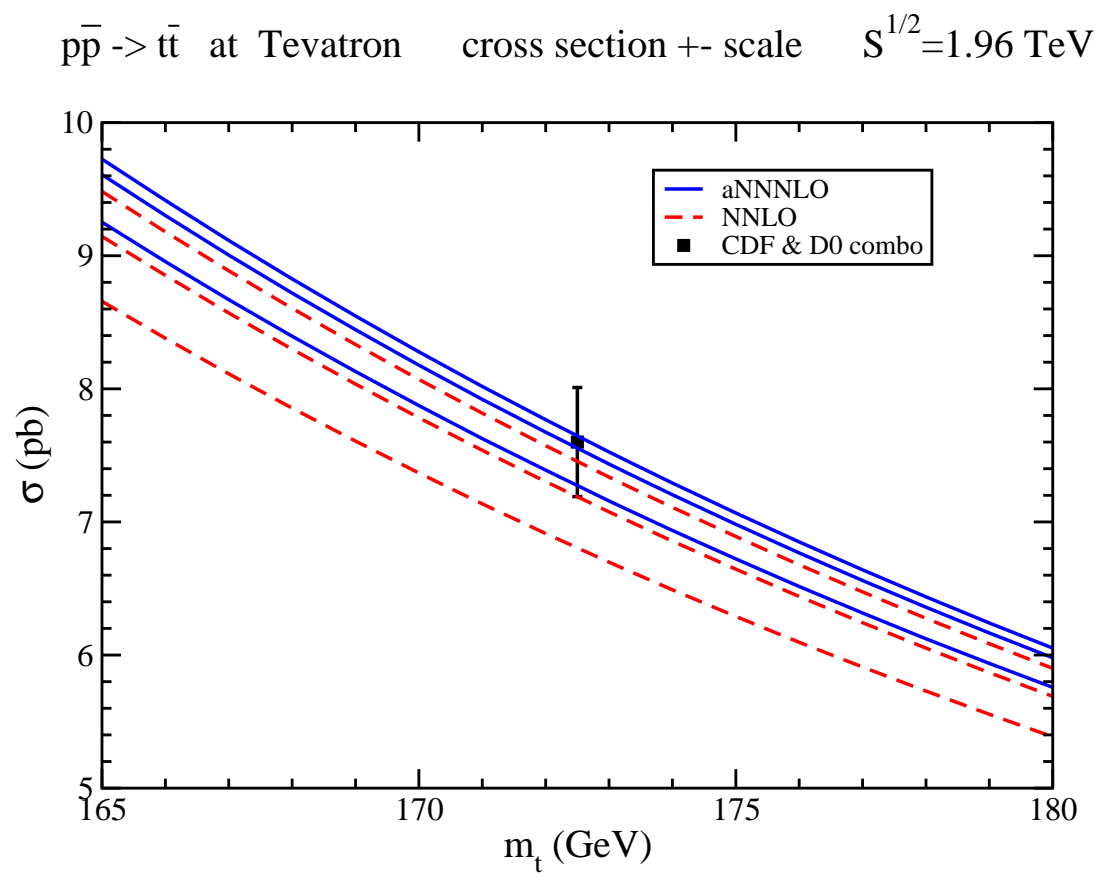

Figure 1: The NNLO and aNNNLO total cross sections for $t \bar{t}$ production at the Tevatron with $\sqrt{S}=1.96 \mathrm{TeV}$ and comparison to CDF and D0 combination data [10]. The central lines at each order are with $\mu=m_{t}$, and the other lines display the upper and lower values from scale variation.

the factorization and renormalization scales, and uncertainties from the parton distributions. We provide more results and discuss these uncertainties in more detail for each collider energy below.

We begin with a study of top-antitop production in proton-antiproton collisions at the Tevatron at $1.96 \mathrm{TeV}$ energy. In Fig. 1] we plot the cross section over a top quark mass range $165 \leq m_{t} \leq 180 \mathrm{GeV}$ at NNLO and aNNNLO. The central result at each order is with a factorization and renormalization scale $\mu=m_{t}$. The independent variation with scales over the interval from $m_{t} / 2$ to $2 m_{t}$ is shown by the upper and lower lines at each order. It is evident that the theoretical scale dependence is smaller at aNNNLO than at NNLO, as expected. The comparison with a recent combination of CDF and D0 Tevatron data [10] shows a nice agreement between theory and experiment. The enhancement from the aNNNLO corrections is significant and improves the description of the Tevatron data.

For a top quark mass of $173.3 \mathrm{GeV}$, the aNNNLO cross section at the Tevatron is

$$
\sigma_{p \bar{p} \rightarrow t \bar{t}}^{\mathrm{aNNNLO}}\left(m_{t}=173.3 \mathrm{GeV}, \sqrt{S}=1.96 \mathrm{TeV}\right)=7.37_{-0.27-0.28}^{+0.09+0.38}=7.37 \pm 0.39 \mathrm{pb} .
$$

Here the first uncertainty is from scale variation over $0.5 \leq \mu / m_{t} \leq 2$ and the second is from the MSTW2008 NNLO pdf errors at 90\% C.L. (to be conservative, we do not use the smaller $68 \%$ C.L. pdf errors). The total uncertainty number is found by adding the scale and pdf errors in quadrature, and it is $\pm 5.3 \%$ at the Tevatron. We also find that the aNNNLO result provides 


\section{$\mathrm{pp}->\mathrm{tt}$ at LHC cross section +- scale $\mathrm{S}^{1 / 2}=7$ and $8 \mathrm{TeV}$}

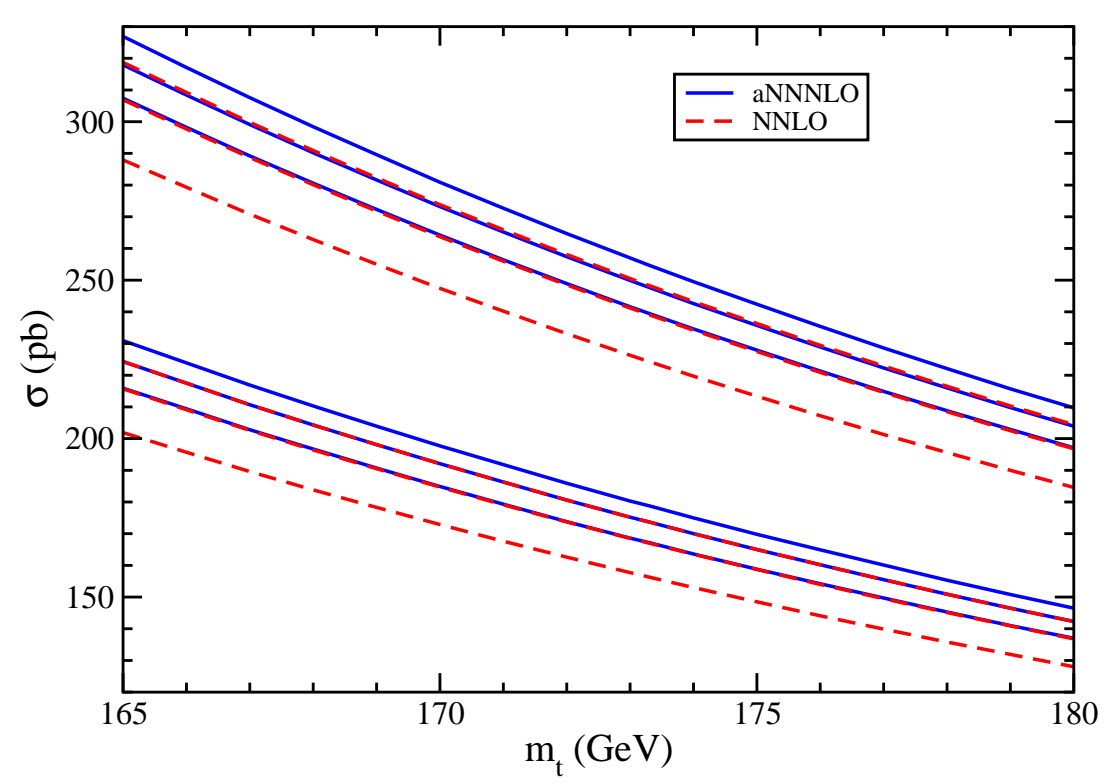

Figure 2: The NNLO and aNNNLO total cross sections for $t \bar{t}$ production at the LHC with $\sqrt{S}=7 \mathrm{TeV}$ (lower lines) and $8 \mathrm{TeV}$ (upper lines). The central lines at each order and each energy are with $\mu=m_{t}$, and the other lines display the upper and lower values from scale variation.

an enhancement of $5.1 \%$ over the NNLO value. Thus both the overall increase of the cross section and the decrease in its uncertainty at aNNNLO relative to NNLO are significant.

We continue with a study of top quark production in proton-proton collisions at the LHC. We present results for the past LHC energies of 7 and $8 \mathrm{TeV}$ and the future LHC energies of 13 and $14 \mathrm{TeV}$.

In Fig. 2 we plot the NNLO and aNNNLO cross sections for top-antitop production at the LHC at $7 \mathrm{TeV}$ and $8 \mathrm{TeV}$ energies over a top quark mass range $165 \leq m_{t} \leq 180 \mathrm{GeV}$. The central results at each order and for each energy are with a factorization and renormalization scale $\mu=m_{t}$, and the scale variation is also displayed. We see that the upper NNLO curves are on top of the central aNNNLO curves at both energies; similarly the central NNLO curves are on top of the lower aNNNLO curves. The overall scale dependence is smaller at aNNNLO.

For a top quark mass of $173.3 \mathrm{GeV}$, the aNNNLO approximate cross section at $7 \mathrm{TeV} \mathrm{LHC}$ energy is

$$
\sigma_{p p \rightarrow t \bar{t}}^{\mathrm{aNNNLO}}\left(m_{t}=173.3 \mathrm{GeV}, \sqrt{S}=7 \mathrm{TeV}\right)=174_{-7-10}^{+5+9}=174_{-12}^{+11} \mathrm{pb}
$$

where the first uncertainty is from scale variation over $0.5 \leq \mu / m_{t} \leq 2$ and the second is from the MSTW2008 NNLO pdf errors at 90\% C.L. The total uncertainty is $+6.2 \%-6.8 \%$. The aNNNLO enhancement over NNLO is $4.0 \%$.

At $8 \mathrm{TeV}$ the corresponding result is

$$
\sigma_{p p \rightarrow t \bar{t}}^{\mathrm{aNNNO}}\left(m_{t}=173.3 \mathrm{GeV}, \sqrt{S}=8 \mathrm{TeV}\right)=248_{-8-13}^{+7+12}=248_{-15}^{+14} \mathrm{pb} .
$$




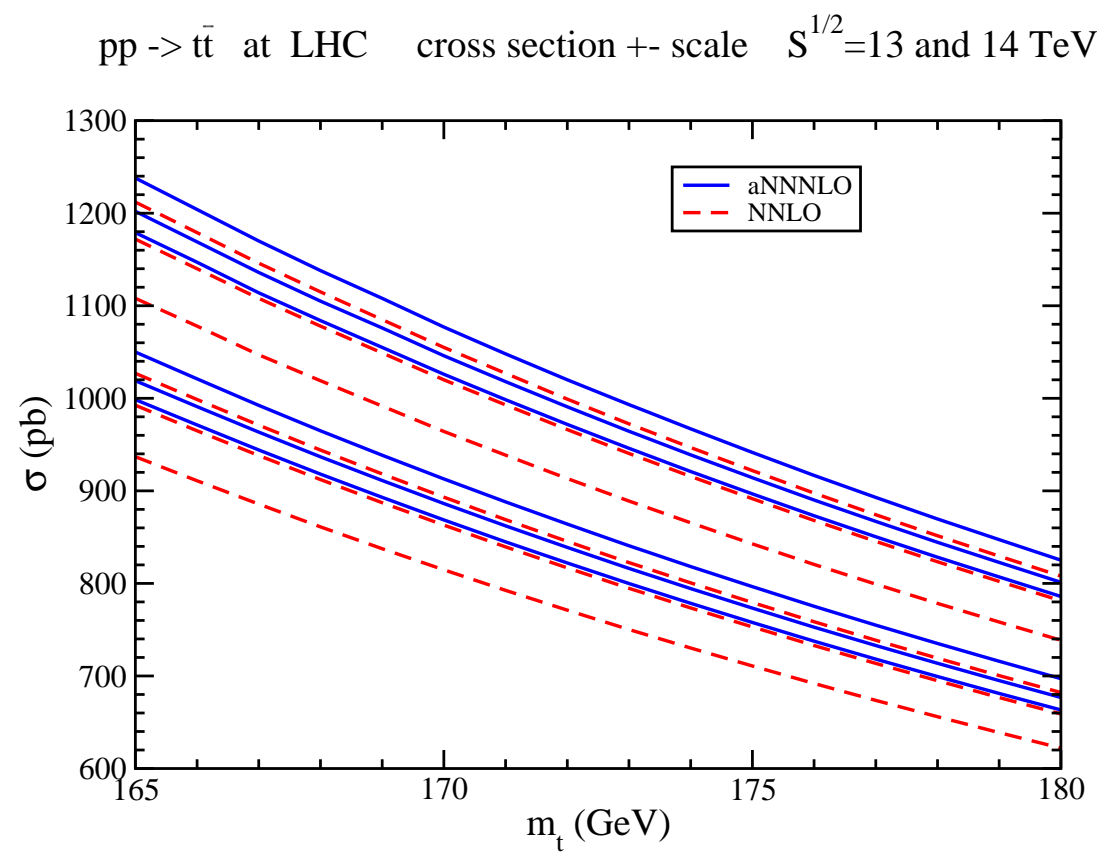

Figure 3: The NNLO and aNNNLO total cross sections for $t \bar{t}$ production at the LHC with $\sqrt{S}=13 \mathrm{TeV}$ (lower lines) and $14 \mathrm{TeV}$ (upper lines). The central lines at each order and each energy are with $\mu=m_{t}$, and the other lines display the upper and lower values from scale variation.

The total uncertainty is $+5.7 \%-6.2 \%$. The aNNNLO enhancement over NNLO is $3.6 \%$.

In Fig. 3 we plot the NNLO and aNNNLO cross sections for top-antitop production at the LHC at $13 \mathrm{TeV}$ and $14 \mathrm{TeV}$ energies over a top quark mass range $165 \leq m_{t} \leq 180 \mathrm{GeV}$. Again the central results at each order and for each energy are with scales $\mu=m_{t}$, and the scale variation is also displayed. Again, the overall scale dependence is smaller at aNNNLO than at NNLO.

The approximate aNNNLO cross section at $13 \mathrm{TeV}$ is

$$
\sigma_{p p \rightarrow t \bar{t}}^{\mathrm{aNNNLO}}\left(m_{t}=173.3 \mathrm{GeV}, \sqrt{S}=13 \mathrm{TeV}\right)=810_{-16-32}^{+24+30}=810_{-36}^{+38} \mathrm{pb} .
$$

The total uncertainty is $+4.8 \%-4.4 \%$ and the aNNNLO enhancement over NNLO is $2.7 \%$ at $13 \mathrm{TeV}$.

The approximate aNNNLO cross section at $14 \mathrm{TeV}$ is

$$
\sigma_{p p \rightarrow t \bar{t}}^{\mathrm{aNNNLO}}\left(m_{t}=173.3 \mathrm{GeV}, \sqrt{S}=14 \mathrm{TeV}\right)=957_{-19-36}^{+28+34}=957_{-41}^{+44} \mathrm{pb} .
$$

The total uncertainty at $14 \mathrm{TeV}$ is $+4.6 \%-4.3 \%$ and the aNNNLO enhancement over NNLO is $2.6 \%$.

It is interesting to discuss the convergence of the perturbative expansion. We write the perturbative series for the cross section through aNNNLO as

$$
\sigma^{\mathrm{aNNNLO}}=\sigma^{(0)}\left[1+\frac{\sigma^{(1)}}{\sigma^{(0)}}+\frac{\sigma^{(2)}}{\sigma^{(0)}}+\frac{\sigma^{(3)}}{\sigma^{(0)}}\right]
$$




\begin{tabular}{|c|c|c|c|c|c|}
\hline \multicolumn{5}{|c|}{ Fractional contributions to the perturbative series for the $t \bar{t}$ cross section } \\
\hline corrections & Tevatron & LHC 7 TeV & LHC 8 TeV & LHC $13 \mathrm{TeV}$ & LHC $14 \mathrm{TeV}$ \\
\hline$\sigma^{(1)} / \sigma^{(0)}$ & 0.236 & 0.470 & 0.476 & 0.493 & 0.496 \\
\hline$\sigma^{(2)} / \sigma^{(0)}$ & 0.106 & 0.178 & 0.177 & 0.172 & 0.170 \\
\hline$\sigma^{(3)} / \sigma^{(0)}$ & 0.068 & 0.066 & 0.059 & 0.045 & 0.043 \\
\hline
\end{tabular}

Table 2: The fractional contributions at higher orders relative to LO, as defined in Eq. (3.6), all calculated with the same NNLO pdf [9], to the $t \bar{t}$ production cross section in $p \bar{p}$ collisions at the Tevatron with $\sqrt{S}=1.96 \mathrm{TeV}$ and in $p p$ collisions at the LHC with $\sqrt{S}=7,8,13$, and $14 \mathrm{TeV}$, with $\mu=m_{t}=173.3 \mathrm{GeV}$.

where $\sigma^{(0)}$ is the LO cross section, $\sigma^{(1)}$ denotes the complete NLO corrections, $\sigma^{(2)}$ denotes the complete NNLO corrections, and $\sigma^{(3)}$ denotes the NNNLO soft-gluon corrections. The values for the fractions $\sigma^{(n)} / \sigma^{(0)}$, with $n=1,2,3$, appear in Table 2 ,

The perturbative series converges slowly. At $14 \mathrm{TeV}, \sigma^{\mathrm{aNNNLO}}=1.709 \sigma^{(0)}$ as can be seen by summing the corresponding entries in Table 2 for Eq. (3.6); the NLO corrections are about half of the LO cross section; the NNLO corrections are about a third of the NLO corrections; and the aNNNLO corrections are about a quarter of the NNLO corrections. Thus the series $\sum_{n=1}^{4} 1 / n !=1.708 \cdots$ approximates the situation very well. If this trend continues in higher orders, which is of course not known but it is amusing to consider the possibility, then using the result $\sum_{n=1}^{\infty} 1 / n !=e-1=1.718 \cdots$ one would expect less than $1 \%$ of the LO cross section (or $0.5 \%$ of the aNNNLO cross section) from additional corrections beyond NNNLO. The above remarks describe the $13 \mathrm{TeV}$ results very well too.

The convergence numbers for 7 and $8 \mathrm{TeV}$ LHC energies are similar though in this case the aNNNLO corrections are about a third of the NNLO corrections, so the trend might me better described by the series $1+(1 / 2) \sum_{n=0}^{2} 1 / 3^{n}$. If this trend continues in higher orders, which again is not known, then using the result $1+(1 / 2) \sum_{n=0}^{\infty} 1 / 3^{n}=7 / 4=1.75$ we again would not expect significant corrections beyond NNNLO.

At the Tevatron, however, the situation is quite different due to the fact that we are closer to threshold. Though the NLO corrections are only about a quarter of the LO cross section (and this is due to the fact that the $g g$ channel is numerically small in this case), the further effect of NNLO and aNNNLO corrections is significant and the convergence is slower. Again, this is due to the fact that we are closer to threshold and it is in agreement with the relatively large cross section measured at the Tevatron. In fact it is clear that as we progress in energy at the LHC from 7 through $14 \mathrm{TeV}$, and thus as we move away from threshold, the NNLO and aNNNLO corrections contribute a progressively smaller fraction to the overall cross section.

It is also important to consider the effects of the order of the pdf being used in the calculation. Since there are no NNNLO pdf available, the aNNNLO cross section that we find using NNLO pdf likely overestimates the result that we would get if we had used NNNLO pdf. It is known that the effect is quite significant when going from NLO pdf to NNLO pdf. For example at $14 \mathrm{TeV}$ LHC energy the NLO cross section using NLO pdf is $885 \mathrm{pb}$, but using NNLO pdf it is $838 \mathrm{pb}$. Although the effect from NNLO pdf to NNNLO pdf should be substantially smaller, it may be comparable to (and partially cancel out) the effect of additional corrections 


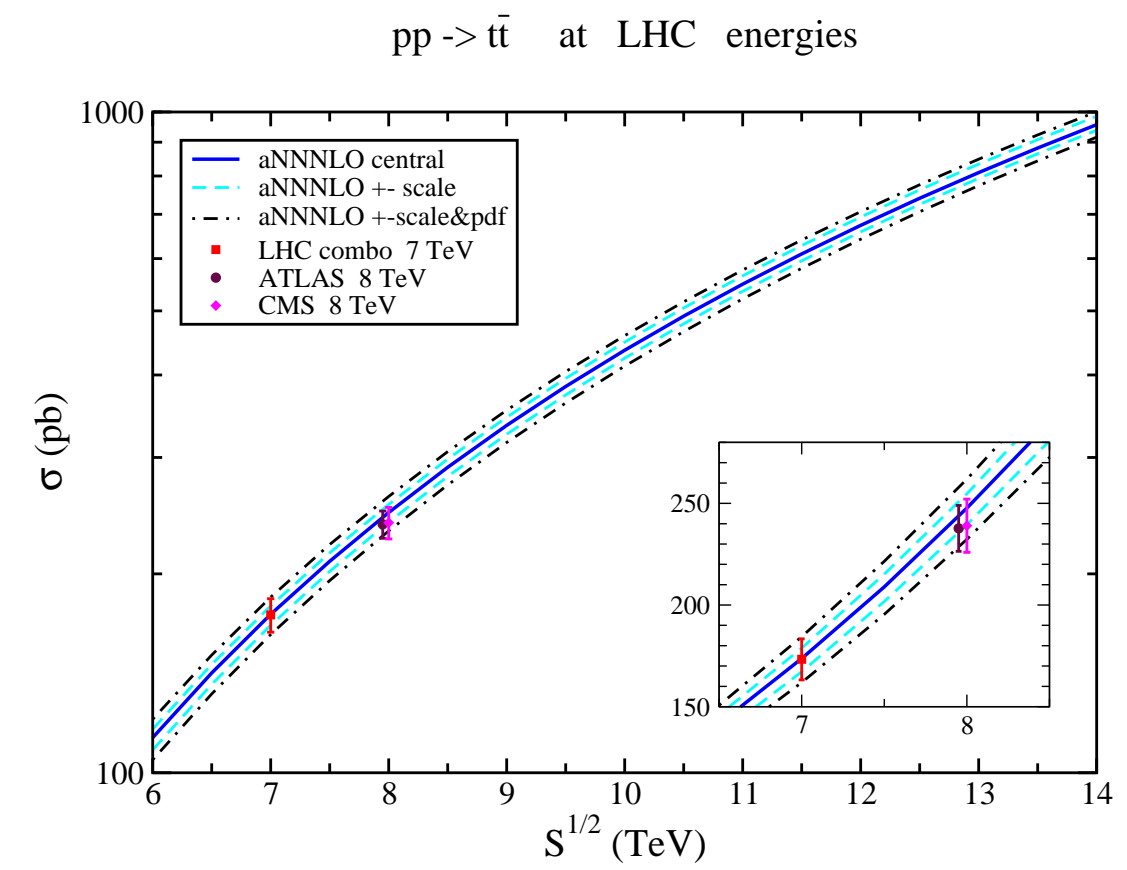

Figure 4: The aNNNLO total cross section with scale and pdf uncertainties for $t \bar{t}$ production at the LHC as a function of collider energy. The inset plot highlights the comparison of theory with ATLAS and CMS data at $7 \mathrm{TeV}$ [11] and $8 \mathrm{TeV}$ [12, 13] LHC energies.

at NNNLO and beyond in the perturbative series.

The above discussions indicate that our aNNNLO results are reliable and accurate, with any additional corrections due to higher orders and higher-order pdf being small and partly canceling out.

In Fig. 4 we show the aNNNLO $t \bar{t}$ cross section as a function of LHC energy. In addition to the central result we show lines indicating the scale variation, as well as upper and lower lines indicating the range from the combined scale and pdf uncertainties. Very good agreement is found with recent ATLAS and CMS data at both $7 \mathrm{TeV}$ [11] and $8 \mathrm{TeV}$ [12, 13] energies. The experimental uncertainties are comparable to the theoretical ones.

We can parameterize the aNNNLO cross sections as functions of the top-quark mass

$$
\sigma^{\mathrm{aNNNLO}}\left(m_{t}\right)=\sigma(173.3) \frac{173.3^{4}}{m_{t}^{4}}\left(1+c_{1} \frac{\left(173.3-m_{t}\right)}{173.3}+c_{2} \frac{\left(173.3-m_{t}\right)^{2}}{173.3^{2}}\right)
$$

and we provide the results for the aNNNLO cross sections at $m_{t}=173.3 \mathrm{GeV}$, denoted as $\sigma(173.3)$, and for the best fit values for the parameters $c_{1}$ and $c_{2}$ to four significant figures in Table 3. The central results are with $\mu=m_{t}$; the results denoted as + scale and - scale are with scale variation between $m_{t} / 2$ and $2 m_{t}$; and the results denoted as "+scale\&pdf" and "-scale\&pdf" give the upper and lower values for the cross section, including scale variation and $90 \%$ C.L. pdf uncertainties added in quadrature. These fits give the aNNNLO cross section with per mille or better accuracy for a large range of top-quark masses, $130 \mathrm{GeV} \leq m_{t} \leq 210$ 


\begin{tabular}{|c|c|c|c|c|c|c|}
\hline \multicolumn{7}{|c|}{ Fit parameters for the aNNNLO $t \bar{t}$ cross section } \\
\hline fit & parameters & Tevatron & LHC 7 TeV & LHC 8 TeV & LHC 13 TeV & LHC 14 TeV \\
\hline \multirow{3}{*}{ central } & $\sigma(173.3)$ & 7.365 & 173.7 & 247.7 & 809.7 & 956.8 \\
& $c_{1}$ & 1.462 & 1.242 & 1.091 & 0.7087 & 0.6564 \\
& $c_{2}$ & 0.9459 & 0.9175 & 0.7429 & 0.2165 & 0.1673 \\
\hline \multirow{4}{*}{ scale } & $\sigma(173.3)$ & 7.455 & 178.8 & 254.7 & 834.0 & 985.4 \\
& $c_{1}$ & 1.469 & 1.249 & 1.099 & 0.7146 & 0.6630 \\
& $c_{2}$ & 0.9690 & 0.9350 & 0.7474 & 0.2264 & 0.1795 \\
\hline \multirow{3}{*}{ scale } & $\sigma(173.3)$ & 7.091 & 167.2 & 239.5 & 793.5 & 938.5 \\
& $c_{1}$ & 1.457 & 1.241 & 1.092 & 0.7085 & 0.6564 \\
& $c_{2}$ & 0.9268 & 0.9192 & 0.7377 & 0.2176 & 0.1673 \\
\hline \multirow{3}{*}{ scale \& pdf } & $\sigma(173.3)$ & 7.754 & 184.4 & 261.9 & 848.3 & 1001.1 \\
& $c_{1}$ & 1.421 & 1.220 & 1.070 & 0.6960 & 0.6451 \\
& $c_{2}$ & 0.8846 & 0.8859 & 0.7313 & 0.2095 & 0.1591 \\
\hline \multirow{3}{*}{ scale \& pdf } & $\sigma(173.3)$ & 6.975 & 161.9 & 232.3 & 773.7 & 915.9 \\
& $c_{1}$ & 1.481 & 1.267 & 1.1157 & 0.7277 & 0.6743 \\
& $c_{2}$ & 0.9774 & 0.9493 & 0.7760 & 0.2346 & 0.1813 \\
\hline
\end{tabular}

Table 3: Values of the fit parameters in Eq. (3.7) for the aNNNLO $t \bar{t}$ production cross section in pb in $p \bar{p}$ collisions at the Tevatron with $\sqrt{S}=1.96 \mathrm{TeV}$ and in $p p$ collisions at the LHC with $\sqrt{S}=7,8,13$, and $14 \mathrm{TeV}$. The fits provide per mille or better accuracy for $130 \mathrm{GeV} \leq m_{t} \leq$ $210 \mathrm{GeV}$.

GeV. The numbers in Tables 1 and 2, in the equations and discussions in the text, and in the figures can all be easily reproduced from these fits.

\section{Conclusions}

We have provided results for aNNNLO total cross sections for $t \bar{t}$ production at the LHC at 7 , 8, 13, and $14 \mathrm{TeV}$ energies and at the Tevatron at $1.96 \mathrm{TeV}$ energy. The NNNLO soft-gluon corrections provide enhancements over the NNLO results which depend on the collider energy and vary from $5.1 \%$ at the Tevatron to $3.6 \%$ at the $8 \mathrm{TeV}$ LHC and $2.6 \%$ at the 14 TeV LHC. Theoretical scale and pdf uncertainties have also been determined. The scale uncertainties are significantly reduced at aNNNLO relative to NNLO. The overall enhancements and uncertainty reductions provided by the aNNNLO corrections enable more accurate and precise theoretical predictions which are also in very good agreement with the latest results from the LHC and the Tevatron. Calculations of aNNNLO top-quark $p_{T}$ and rapidity distributions are also possible in our double-differential resummation formalism and will appear in the future. 


\section{Acknowledgements}

This material is based upon work supported by the National Science Foundation under Grant No. PHY 1212472.

\section{References}

[1] P. Nason, S. Dawson, and R.K. Ellis, Nucl. Phys. B 303, 607 (1988); Nucl. Phys. 327, 49 (1989); (E) B 335, 260 (1990).

[2] W. Beenakker, H. Kuijf, W.L. van Neerven, and J. Smith, Phys. Rev. D 40, 54 (1989).

[3] W. Beenakker, W.L. van Neerven, R. Meng, G.A. Schuler, and J. Smith, Nucl. Phys. B 351, 507 (1991).

[4] N. Kidonakis and B.D. Pecjak, Eur. Phys. J. C 72, 2084 (2012) [arXiv:1108.6063 [hep-ph]].

[5] M. Czakon, P. Fiedler, and A. Mitov, Phys. Rev. Lett. 110, 252004 (2013) arXiv:1303.6254 [hep-ph]].

[6] N. Kidonakis, Phys. Rev. D 82, 114030 (2010) [arXiv:1009.4935 [hep-ph]].

[7] N. Kidonakis, in Proceedings of HQ2013 Summer School, arXiv:1311.0283 [hep-ph].

[8] N. Kidonakis, Phys. Rev. D 73, 034001 (2006) [hep-ph/0509079].

[9] A.D. Martin, W.J. Stirling, R.S. Thorne, and G. Watt, Eur. Phys. J. C 63, 189 (2009) arXiv:0901.0002 [hep-ph]].

[10] CDF and D0 Collaborations, Phys. Rev. D 89, 072001 (2014) [arXiv:1309.7570 [hep-ex]].

[11] ATLAS and CMS Collaborations, ATLAS-CONF-2012-134, CMS-PAS-TOP-12-003.

[12] ATLAS Collaboration, ATLAS-CONF-2013-097.

[13] CMS Collaboration, JHEP 02, 024 (2014) [arXiv:1312.7582 [hep-ex]]. 